\title{
Elevated preoperative aspartate aminotransferase to lymphocyte ratio index as an independent prognostic factor for patients with hepatocellular carcinoma after hepatic resection
}

\author{
Junfei Jin ${ }^{1,2, *}$, Pengpeng Zhu ${ }^{1, *}$, Yan Liao ${ }^{3}$, Jun Li ${ }^{1}$, Weijia Liao ${ }^{1,2}$ and Songqing $\mathrm{He}^{1,2}$ \\ ${ }^{1}$ Laboratory of Hepatobiliary and Pancreatic Surgery, Affiliated Hospital of Guilin Medical University, Guilin, Guangxi, People's \\ Republic of China \\ ${ }^{2}$ Guangxi Key Laboratory of Molecular Medicine in Liver Injury and Repair, Guilin Medical University, Guilin, Guangxi, People's \\ Republic of China \\ ${ }^{3}$ Disease Prevention and Control Center of Guilin, Guilin, Guangxi, People's Republic of China \\ * These authors have contributed equally to this work \\ Correspondence to: Songqing He, email: dr_hesongqing@163.com \\ Weijia Liao, email: liao288@163.com \\ Keywords: oncology, hepatocellular carcinoma, treatment, prognosis, therapy \\ Received: March 17, $2015 \quad$ Accepted: May 13, $2015 \quad$ Published: May 25, 2015
}

This is an open-access article distributed under the terms of the Creative Commons Attribution License, which permits unrestricted use, distribution, and reproduction in any medium, provided the original author and source are credited.

\section{ABSTRACT}

Few studies have elucidated the relationship between preoperative aspartate aminotransferase (AST) to lymphocyte ratio and high incidence of hepatocellular carcinoma (HCC). In search of a simple non-invasive prognostic marker, we investigated the prognostic significance of AST to lymphocyte ratio index (ALRI) in HCC. We reviewed retrospectively clinical parameters of 371 HCC patients who were treated with hepatectomy. Receiver operating characteristic (ROC) curve analysis was performed to determine the cut-off value of preoperative ALRI. The predictive value of preoperative ALRI in HCC was evaluated by univariate and multivariate analyses using Cox proportional hazards regression modeling, and the survival probability of HCC patients was acquired by the Kaplan-Meier plots. In addition, stratified analysis was used to investigate the impact of preoperative ALRI on survival in different HCC subgroups. The results showed that preoperative ALRI was closely correlated with age $(p=0.007)$, median size $(p=0.004)$, clinical tumor-node-metastasis (TNM) stage $(p<0.001)$, and portal vein tumor thrombosis (PVTT) $(p<0.001)$. Survival analysis indicated that HCC patients with preoperative ALRI > $\mathbf{2 5 . 2}$ have a poorer disease-free survival (DFS) and overall survival (OS) after tumor resection. Multivariate analysis further identified preoperative ALRI $>25.2(p=0.002)$, III-IV of TNM stage $(p=$ $0.011)$, PVTT $(p=0.035)$, size of tumor $>5 \mathrm{~cm}(p<0.001)$ as independent risk factors of DFS; and preoperative ALRI $>25.2(p=0.001)$, III-IV of TNM stage $(p=$ $0.005)$, PVTT $(p=0.012)$, size of tumor $>5 \mathrm{~cm}(p<0.001)$, recurrence $(p<0.001)$ as independent prognostic factors for OS in HCC patients. Additionally, preoperative ALRI also showed different prognostic value in various subgroups of HCC. Elevated preoperative ALRI as a noninvasive, simple, and easily assessable parameter is an independent effective predictor of prognosis for patients with HCC.

\section{INTRODUCTION}

Hepatocellular carcinoma (HCC) is the fifth most lethal malignant cancer and the third most frequent cause of cancer mortality worldwide [1]. The annual incidence has increased in recent years. In the year 2002, China had 55\% of the world's diagnosed HCC patients, making this disease a burden on the country [2]. Despite the sophisticated progress in diagnostic techniques and advanced instruments in surgery, the prognosis remained 
far from satisfactory due to the high rate of recurrence and metastasis $[3,4]$. The lack of a sensitive clinical parameter has a profound impact on HCC therapeutic practice. Therefore, searching for an effective preoperative marker is important in diagnosis and prognosis of HCC; it is also beneficial to HCC patients' treatment.

In clinical practice, doctors commonly use liver enzyme aspartate aminotransferase (AST), which may reflect liver function or damage, as a routine biochemical test for diagnosis of various diseases including HCC $[5,6]$. Growing evidence demonstrates the relationship between high serum levels of AST and mortalities of HCC [7,8 ].

There is a highly significant relationship between systemic chronic inflammatory disorders and high incidence of a variety of cancers including HCC [912]. Malignant tumor cells lead to an intracellular inflammatory program, which may result in the promotion of angiogenesis and the inhibition of apoptosis, ultimately, causing tumor formation, proliferation, metastasis, and recurrence [13, 14]. Emerging evidence indicates that patrolling and infiltrating lymphocytes reflect the patients' inflammatory status as well as their bodies' ability to exert decisive function in antitumor immune responses [15-17].

To our knowledge, no population-based study has been conducted related to the prognostic value of preoperative AST to lymphocyte ratio index (ALRI) in predicting tumor recurrence in HCC patients after curative resection. An improved understanding the value of preoperative ALRI in HCC survival will provoke new ideas for therapeutic approaches.

A

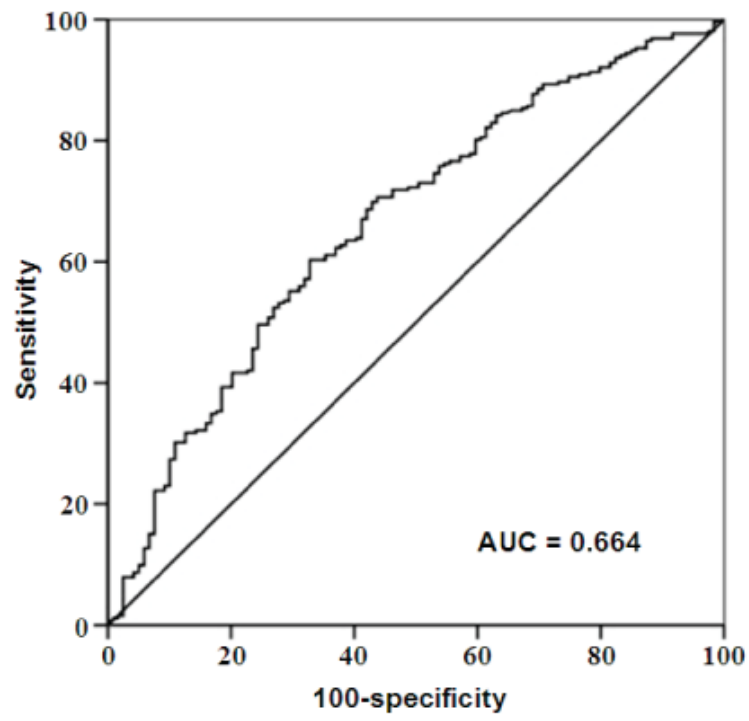

\section{RESULTS}

\section{Determination of the cutoff value of preoperative ALRI in HCC}

To identify the optimal cut-off value of preoperative ALRI, an ROC curve was obtained by using MedCalc analysis, which indicated that the score of 25.2 has the maximum sensitivity and specificity for predicting survival status. The area under receiver operating curves (AUC) was 0.664 with a $95 \%$ CI of 0.613 to 0.712 , a sensitivity of $60.3 \%$, and a specificity of $67.2 \%$ (Figure $1 \mathrm{~A}$ ).

\section{Stratified analysis according to HBV infection, TNM stage, and clinical PVTT}

Patients were stratified according to HBV infection, TNM stage, and clinical PVTT in order to compare the preoperative ALRI in two different HCC subgroups. We found that when $\mathrm{HBV}$ infection was present in $\mathrm{HCC}$ patients, preoperative ALRI was significantly higher compared to those without HBV infection (39.14 \pm 1.967 , $27.48 \pm 3.010$, respectively) ( $t=2.475, p=0.0138$, Figure 1B). This tendency was also found in HCC patients with III-IV of TNM stage in contrast to those with I-II of TNM stage (44.86 $\pm 2.591,27.94 \pm 1.967$, respectively) $(t=$ $5.006, p<0.0001$, Figure 1B). In addition, the preoperative ALRI in HCC patients with PVTT increases significantly compared to those without PVTT $(49.13 \pm 3.911,33.32$
B

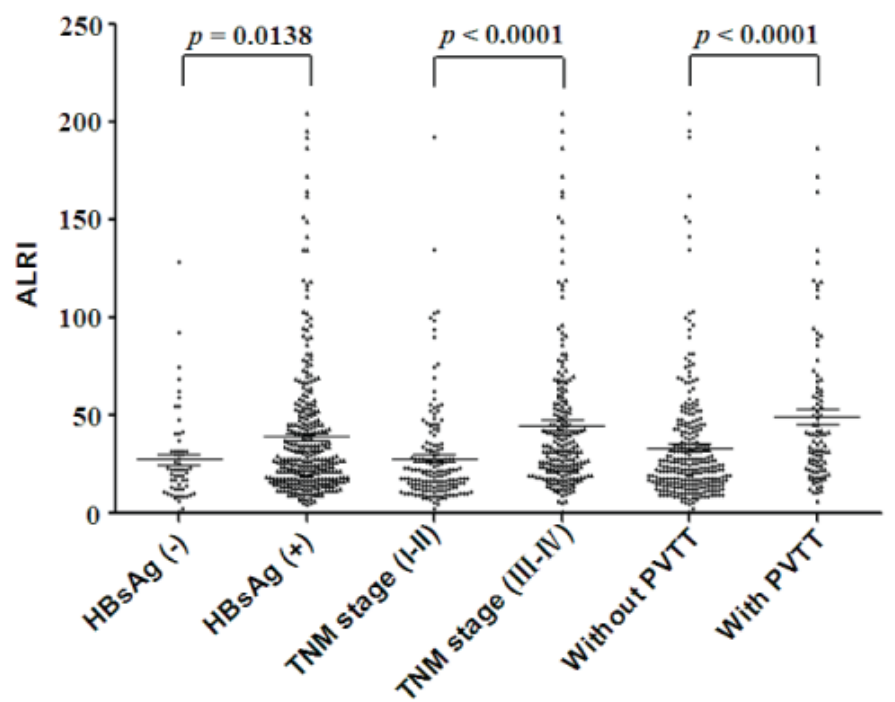

Figure 1: ROC curve, and stratified analysis of preoperative ALRI in HCC subgroups. A. Receiver operating characteristic (ROC) analysis was performed to evaluate the prognostic value of preoperative ALRI. The area under the ROC curve value was 0.664 . B. All 371 cases of HCC patients were stratified based on HBV Infection, TNM stage, and clinical PVTT, thus comparing preoperative ALRI in different HCC subgroups. The proportions of patients with elevated preoperative ALRI along with HBV infection, III-IV of TNM stage, and clinical PVTT are much higher than those without HBV infection, I-II of TNM stage, and without PVTT, respectively $(p<0.05)$. 
Table 1: Correlation between the clinicopathologic variables and ALRI in HCC.

\begin{tabular}{|c|c|c|c|c|c|c|}
\hline \multirow{2}{*}{ Clinical character } & \multirow{2}{*}{ variable } & \multirow{2}{*}{$\begin{array}{l}\text { No.of } \\
\text { patients }\end{array}$} & \multicolumn{2}{|c|}{ ALRI } & \multirow{2}{*}{$\chi^{2}$} & \multirow{2}{*}{$p$ value } \\
\hline & & & $\leq 25.2$ & $>25.2$ & & \\
\hline \multirow[t]{2}{*}{ Age (years) } & $\leq 50$ & 201 & $105(52.2)$ & $96(47.8)$ & 7.275 & 0.007 \\
\hline & $>50$ & 170 & $65(38.2)$ & $105(61.8)$ & & \\
\hline \multirow[t]{2}{*}{ Gender } & Female & 48 & $23(47.9)$ & $25(52.1)$ & 0.097 & 0.755 \\
\hline & Male & 323 & $147(45.5)$ & $176(54.5)$ & & \\
\hline \multirow[t]{2}{*}{ Family history } & No & 319 & $147(46.1)$ & $172(53.9)$ & 0.062 & 0.804 \\
\hline & Yes & 52 & $23(44.2)$ & $29(55.8)$ & & \\
\hline \multirow[t]{2}{*}{ HBsAg } & Negative & 59 & $28(47.5)$ & $31(52.5)$ & 0.076 & 0.783 \\
\hline & Positive & 312 & $142(45.5)$ & $170(54.5)$ & & \\
\hline \multirow[t]{2}{*}{$\operatorname{AFP}(\mu \mathrm{g} / 1)$} & $\leq 20$ & 97 & $50(51.5)$ & $47(48.5)$ & 1.734 & 0.188 \\
\hline & $>20$ & 274 & $120(43.8)$ & $154(56.2)$ & & \\
\hline \multirow[t]{2}{*}{ Median size $(\mathrm{cm})$} & $\leq 5$ & 124 & $70(56.5)$ & $54(43.5)$ & 8.477 & 0.004 \\
\hline & $>5$ & 247 & $100(40.5)$ & $147(59.5)$ & & \\
\hline \multirow[t]{2}{*}{ Cirrhosis } & No & 33 & $18(54.5)$ & $15(45.5)$ & 1.110 & 0.292 \\
\hline & Yes & 338 & $152(45.0)$ & $186(55.0)$ & & \\
\hline \multirow[t]{2}{*}{ Tumor number } & Single & 248 & $117(47.2)$ & $131(52.8)$ & 0.553 & 0.457 \\
\hline & Multiple & 123 & $53(43.1)$ & $70(56.9)$ & & \\
\hline \multirow[t]{2}{*}{ TNM stage } & $\mathrm{I}-\mathrm{II}$ & 166 & $102(61.4)$ & $64(38.6)$ & 29.539 & $<0.001$ \\
\hline & III-IV & 205 & $68(33.2)$ & $137(66.8)$ & & \\
\hline \multirow[t]{2}{*}{ PVTT } & No & 278 & $144(51.8)$ & $134(48.2)$ & 15.956 & $<0.001$ \\
\hline & Yes & 93 & $26(28.0)$ & $67(72.0)$ & & \\
\hline \multirow[t]{2}{*}{ Distant metastasis } & No & 342 & $160(46.8)$ & $182(53.2)$ & 1.629 & 0.202 \\
\hline & Yes & 29 & $10(34.5)$ & $19(65.5)$ & & \\
\hline \multirow[t]{2}{*}{ Recurrence } & No & 238 & $101(42.4)$ & $137(57.6)$ & 3.064 & 0.080 \\
\hline & Yes & 133 & $69(51.9)$ & $64(48.1)$ & & \\
\hline
\end{tabular}

ALRI, aspartate aminotransferase to lymphocyte ratio index; HBsAg, hepatitis B surface antigen; AFP, alpha-fetoprotein; TNM, tumor-node-metastasis; PVTT, portal vein tumor thrombus.
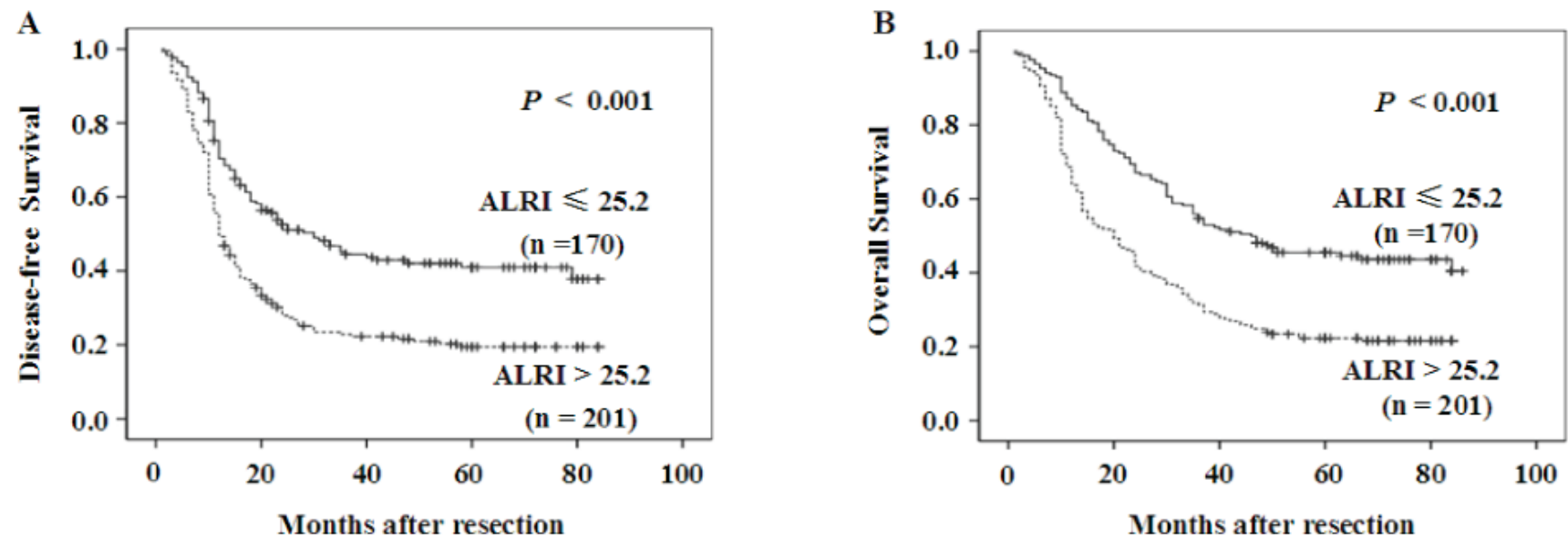

Figure 2: Kaplan-Meier survival curves of HCC patients after hepatectomy. Patients were divided into two groups: ALRI $\leq$ 25.2 and $>25.2$ by optimal cutoff value of ALRI. DFS in patients with ALRI $>25.2$ was shorter than those with ALRI $\leq 25.2$ (Figure A). OS in patients with ALRI $>25.2$ was shorter than those with ALRI $\leq 25.2$ (Figure B). 
Table 2: Association between ALRI, clinical parameters and disease-free survival/overall survival.

\begin{tabular}{|c|c|c|c|c|c|c|c|c|}
\hline \multirow{2}{*}{ Clinical character } & \multirow{2}{*}{ Category } & \multirow{2}{*}{$\begin{array}{l}\text { No.of } \\
\text { patients }\end{array}$} & \multicolumn{3}{|c|}{ Disease-free survival (months) } & \multicolumn{3}{|c|}{ Overall survival (months) } \\
\hline & & & Mean & $95 \% \mathrm{CI}$ & $p$ value & Mean & $95 \% \mathrm{CI}$ & $p$ value \\
\hline \multirow[t]{2}{*}{ ALRI } & $\leq 25.2$ & 170 & 44.19 & $38.89-49.50$ & $<0.001$ & 51.22 & $46.31-56.12$ & $<0.001$ \\
\hline & $>25.2$ & 201 & 27.32 & $23.17-31.48$ & & 32.80 & $28.74-36.86$ & \\
\hline \multirow[t]{2}{*}{ Age (years) } & $\leq 50$ & 201 & 35.07 & $30.45-39.70$ & 0.905 & 40.91 & $36.41-45.42$ & 0.773 \\
\hline & $>50$ & 170 & 34.96 & $29.88-40.04$ & & 41.48 & $36.70-46.26$ & \\
\hline \multirow[t]{2}{*}{ Gender } & Female & 48 & 44.76 & $34.26-55.27$ & 0.059 & 50.38 & $40.95-59.81$ & 0.029 \\
\hline & Male & 323 & 33.72 & $30.13-37.30$ & & 40.01 & $36.51-43.50$ & \\
\hline \multirow[t]{2}{*}{ Family history } & No & 319 & 33.63 & $30.02-37.25$ & 0.065 & 40.31 & $36.80-43.83$ & 0.093 \\
\hline & Yes & 52 & 43.52 & $33.71-53.33$ & & 47.52 & $38.36-56.68$ & \\
\hline \multirow[t]{2}{*}{ HBsAg } & Negative & 59 & 37.35 & $28.68-46.02$ & 0.356 & 43.84 & $35.52-52.15$ & 0.491 \\
\hline & Positive & 312 & 34.53 & $30.80-38.25$ & & 40.42 & $36.90-43.95$ & \\
\hline \multirow[t]{2}{*}{$\operatorname{AFP}(n g / m L)$} & $\leq 20$ & 97 & 38.17 & $31.36-44.98$ & 0.207 & 44.99 & $38.82-51.16$ & 0.133 \\
\hline & $>20$ & 274 & 33.89 & $29.95-37.84$ & & 39.96 & $36.10-43.82$ & \\
\hline \multirow[t]{2}{*}{ Tumor size $(\mathrm{cm})$} & $\leq 5$ & 124 & 53.27 & $47.21-59.33$ & $<0.001$ & 59.20 & $53.92-64.49$ & $<0.001$ \\
\hline & $>5$ & 247 & 25.92 & $22.29-29.54$ & & 32.04 & $28.40-35.68$ & \\
\hline \multirow[t]{2}{*}{ Cirrhosis } & No & 33 & 32.62 & $21.27-43.97$ & 0.411 & 39.56 & $28.81-50.30$ & 0.638 \\
\hline & Yes & 338 & 35.28 & $31.69-38.87$ & & 41.01 & $37.62-44.39$ & \\
\hline \multirow[t]{2}{*}{ Tumor number } & Single & 248 & 39.73 & $35.41-44.05$ & $<0.001$ & 46.50 & $42.42-50.58$ & $<0.001$ \\
\hline & Multiple & 123 & 25.40 & $20.30-30.50$ & & 30.94 & $25.85-36.03$ & \\
\hline \multirow[t]{2}{*}{ TNM stage } & I-II & 166 & 49.12 & $43.76-54.48$ & $<0.001$ & 57.00 & $52.28-61.72$ & $<0.001$ \\
\hline & III-IV & 205 & 23.70 & $19.96-27.45$ & & 28.56 & $24.82-32.20$ & \\
\hline \multirow[t]{2}{*}{ PVTT } & No & 278 & 40.47 & $36.34-44.59$ & $<0.001$ & 47.85 & $44.01-51.70$ & $<0.001$ \\
\hline & Yes & 93 & 19.31 & $14.71-23.91$ & & 22.16 & $17.57-26.75$ & \\
\hline \multirow[t]{2}{*}{ Distant metastasis } & No & 342 & 36.00 & $32.39-39.61$ & 0.044 & 42.50 & $39.03-45.97$ & 0.016 \\
\hline & Yes & 29 & 22.96 & $15.11-30.80$ & & 29.41 & $19.66-39.17$ & \\
\hline \multirow[t]{2}{*}{ Recurrence } & No & 238 & & & & 34.95 & $30.71-39.19$ & $<0.001$ \\
\hline & Yes & 133 & & & & 52.36 & $47.88-56.84$ & \\
\hline
\end{tabular}

ALRI, aspartate aminotransferase to lymphocyte ratio index; HBsAg, hepatitis B surface antigen; AFP, alpha-fetoprotein; TNM, tumor-node-metastasis; PVTT, portal vein tumor thrombus; CI, confidence interval.

\pm 1.854 , respectively) $(t=4.030, p<0.0001$, Figure 1B). The data showed here demonstrated that preoperative ALRI has a strong connection with HBV infection, latestage of HCC, and hepatic metastases.

\section{Association between preoperative ALRI and clinicopathological features}

The relationship between preoperative ALRI and clinicopathological variables of patients with HCC was investigated after the results obtained from the ROC 
Table 3: Cox multivariate proportional hazard model of independent predictors on disease-free and overall survival.

\begin{tabular}{lll}
\hline \multicolumn{1}{c}{ Variable } & Hazard ratio (95\% CI) & $P$ value \\
\hline Disease-free survival & & \\
\hline ALRI ( $>25.2 v s \leq 25.2)$ & $1.512(1.163-1.966)$ & $\mathbf{0 . 0 0 2}$ \\
Tumor number (multiple $v s$ single) & $1.127(0.857-1.481)$ & 0.393 \\
TNM stage (III-IV $v s$ I-II) & $1.536(1.103-2.138)$ & $\mathbf{0 . 0 1 1}$ \\
PVTT (yes $v s$ no) & $1.386(1.024-1.875)$ & $\mathbf{0 . 0 3 5}$ \\
Tumor size, cm (>5 vs $\leq 5$ ) & $2.051(1.456-2.887)$ & $<\mathbf{0 . 0 0 1}$ \\
Distant metastasis (yes $v s$ no) & $1.190(0.779-1.816)$ & 0.421 \\
Overall survival & & \\
ALRI ( $>25.2 v s \leq 25.2)$ & $1.552(1.192-2.020)$ & $\mathbf{0 . 0 0 1}$ \\
Tumor number (multiple $v s$ single) & $1.130(0.858-1.487)$ & 0.385 \\
TNM stage (III-IV $v s$ I-II) & $1.612(1.157-2.246)$ & $\mathbf{0 . 0 0 5}$ \\
PVTT (yes $v s$ no) & $1.494(1.094-2.039)$ & $\mathbf{0 . 0 1 2}$ \\
Tumor size, cm $(>5 v s \leq 5$ ) & $1.955(1.388-2.753)$ & $<\mathbf{0 . 0 0 1}$ \\
Distant metastasis (yes $v s$ no) & $1.386(0.903-2.127)$ & 0.135 \\
Gender (male $v s$ female) & $1.390(0.910-2.123)$ & 0.127 \\
Recurrence (yes $v s$ no) & $1.647(1.253-2.165)$ & $<\mathbf{0 . 0 0 1}$ \\
\hline ALRI, & &
\end{tabular}

ALRI, aspartate aminotransferase to lymphocyte ratio index; TNM, tumor-node-metastasis; PVTT, portal vein tumor thrombus.

curve were analyzed. The data showed that preoperative ALRI was correlated with age $\left(\chi^{2}=7.275, p=0.007\right)$, median size $\left(\chi^{2}=8.477, p=0.004\right)$, clinical TNM stage $\left(\chi^{2}=29.539, p<0.001\right)$, and PVTT $\left(\chi^{2}=15.956, p<\right.$ $0.001)$. Nonetheless, there were no statistical connections between preoperative ALRI and other clinicopathological parameters including gender, family history, HBsAg, AFP, cirrhosis, tumor number, distant metastasis, and recurrence (all $p>0.05$, Table 1).

\section{The correlation between the survival status and preoperative ALRI}

To determine the prognostic value of preoperative ALRI in postsurgical HCC patients, Kaplan-Meier survival analysis was conducted. The median DFS time in HCC patients with preoperative ALRI > 25.2 was 27.32 months, which was significantly shorter than that in patients with preoperative ALRI $\leq 25.2$ (44.19 months) $(p$ $<0.001$, Figure 2A). Furthermore, the median OS time in the group of preoperative ALRI > 25.2 was 32.80 months, which was remarkably shorter than that in the group of the preoperative ALRI $\leq 25.2$ (51.22 months) $(p<0.001$, Figure 2B).

\section{Univariate analysis of prognostic variables in HCC patients}

The results from univariate analysis revealed that preoperative ALRI $>25.2(p<0.001)$, size of tumor $>5$ $\mathrm{cm}(p<0.001)$, multiple tumor number $(p<0.001)$, III-IV of TNM stage $(p<0.001)$, PVTT $(p<0.001)$, and distant metastasis $(p=0.044)$ were responsible for the DFS of HCC patients. Significant predictors of OS in patients with HCC after resection were preoperative ALRI $>25.2(p<$ $0.001)$, male $(p=0.029)$, size of tumor $>5 \mathrm{~cm}(p<0.001)$, multiple tumor number $(p<0.001)$, III-IV of TNM stage $(p<0.001)$, PVTT $(p<0.001)$, distant metastasis $(p=$ $0.016)$, and recurrence $(p<0.001)$ (Table 2$)$. 

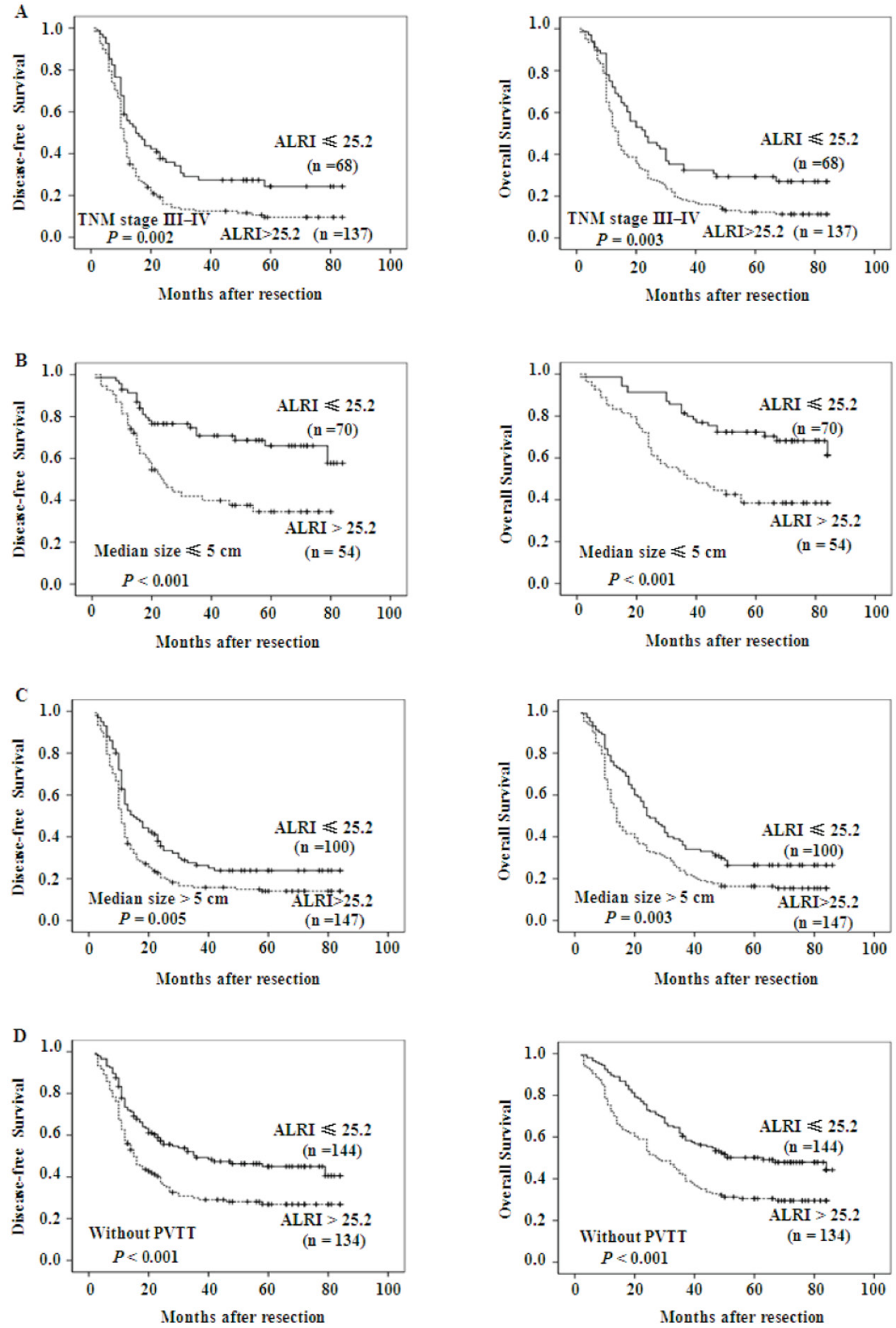

Figure 3: Kaplan-Meier survival curves of different HCC subgroups after hepatectomy. Kaplan-Meier survival estimates and log-rank tests were used to analyze the prognostic significance of preoperative ALRI in each subgroup. ALRI > 25.2 significantly correlated with shorter DFS and OS in subgroups with TNM stage of III/IV (Figure A), tumor size $\leq 5 \mathrm{~cm}$ (Figure B), tumor size $>5 \mathrm{~cm}$ (Figure C), and those without PVTT (Figure D). 


\section{Multivariate analysis of prognostic variables in HCC patients}

The multivariate Cox's proportional hazard regression analysis was used to find the best predictors of the survival of postsurgical HCC patients. The results revealed that preoperative ALRI > $25.2(\mathrm{HR}, 1.512 ; 95 \%$ CI, $1.163-1.966 ; p=0.002)$, III-IV of TNM stage (HR, $1.536 ; 95 \% \mathrm{CI}, 1.103-2.138 ; p=0.011)$, PVTT (HR, $1.386 ; 95 \%$ CI, $1.024-1.875 ; p=0.035)$, and size of tumor $>5 \mathrm{~cm}$ (HR, 2.051; 95\% CI, 1.456-2.887; $p<0.001)$ were independent prognostic markers for DFS among the HCC cohort. The preoperative ALRI > 25.2 (HR, 1.552; 95\% CI, 1.192-2.020; $p=0.001)$, III-IV of TNM stage (HR, 1.612; 95\% CI, 1.157-2.246; $p=0.005)$, PVTT (HR, $1.494 ; 95 \%$ CI, 1.094-2.039; $p=0.012)$, size of tumor $>$ $5 \mathrm{~cm}$ (HR, 1.955; 95\% CI, 1.388-2.753; $p<0.001)$, and recurrence (HR, 1.647; 95\% CI, 1.253-2.165; $p<0.001)$ were independent predictors of OS in patients with HCC (Table 3).

\section{Prognostic values of preoperative ALRI in different HCC subgroups}

The data above confirmed that preoperative ALRI $>25.2$ was significantly correlated with shorter DFS ( $p$ $<0.001)$ and OS $(p<0.001)$ of HCC patients. We further evaluated the prognostic value of preoperative ALRI in different subgroups of HCC patients. The results showed that preoperative ALRI was a prognostic indicator for DFS $(p=0.002)$ and OS $(p=0.003)$ in patients with TNM stage of III-IV (Figure 3A). Additionally, in the subgroup of tumor size $\leq 5 \mathrm{~cm}$, preoperative ALRI $>25.2$ appeared apparent prognostic value in predicting poorer DFS and OS (Figure 3B; $p<0.001, p<0.001$, respectively), and this prognostic value also existed in subgroup with tumor size $>5 \mathrm{~cm}$ (Figure 3C; $p=0.005, p=0.003$, respectively) or in patients without PVTT (Figure 3D). Therefore, these data suggested that preoperative ALRI was more sensitive to predict the prognosis of HCC patients than other clinical parameters, which appeared a promising prognostic value in various HCC subgroups whose survival is difficult to be predicted.

\section{DISCUSSION}

The prediction of prognosis plays a key role in therapeutic options of HCC. A huge endeavor had been made in searching of valid indicators predicting $\mathrm{HCC}$ prognosis, but clinically doctors still rely on conventional pathological diagnosis such as tumor size, TNM stage, and distant metastasis status. Therefore, finding a non-invasive biochemical marker is of great importance in patients with HCC.

AST is a biochemical enzyme that plays a crucial role in the metabolism of amino acid and exists mainly in liver but as well as in heart, skeletal muscle, kidney, and brain; it is well known that AST is a reliable and sensitive biochemical marker of liver injury. A higher AST level is correlated with a greater influx of hepatitis B virus, that associates with decreased overall survival in HCC patients [8]. However, advancing liver diseases may be related to mitochondrial injury, which leads the release of AST to the mitochondria as well as the cytoplasm of hepatocytes [18]. Additionally, some studies have found that AST-to-ALT ratio was an excellent predictor for a large number of hepatic disorders [19-21] and classical aspartate aminotransferase to platelet ratio index (APRI) was associated witha poor prognosis in liver diseases including HCC [22-25].

The close correlation between systemic inflammation and malignant neoplasm is all-too-common in the liver because HCC almost exclusively arises due to chronic hepatitis disease [26]. Lymphocyte plays central roles in host' antitumor immune responses, and its presence closely relates to a reduced risk of relapse in several situations [27]. Systemic inflammatory cytokines, such as tumor necrosis factor $\alpha(\mathrm{TNF} \alpha)$, interferon $\gamma(\mathrm{IFN} \gamma)$, and Toll-like receptor 3 ligands, stimulate the expression of a series of intra-tumor chemokines. These chemokines include chemokine (C-C motif) ligand 2 (CCL2), CCL5, and chemokine (C-X-C motif) ligand 10 (CXCL10), which have a close relationship with the increase of $\mathrm{CD}^{+} \mathrm{T}$ helper type 1 lymphocytes (Th1) cells and $\mathrm{CD} 8^{+}$cytotoxic $\mathrm{T}$ lymphocytes (CTLs). Once activated, the CD4 ${ }^{+} \mathrm{Th} 1$ cells will eliminate malignant tumor cells and effectively prevent oncogenesis [28, 29]. The initiated CTLs will directly kill carcinoma cells or inhibit angiogenesis by secreting cytotoxin [30]. Some evidence suggests that CTLs located in the special microenvironment are positively relevant to favorable prognosis in numerous malignant cancers [31]. Conversely, as a subpopulation of T lymphocytes, natural killer T (NKT) cells making up about $30 \%$ of all lymphocytes in the liver [32] may also act as an important role in antitumor immunity. It has been reported that NKT lymphocytes can perform antimetastatic roles by stimulating IL-12 [15]. Moreover, once activated by NKT cells, NK cells perform the main functions in clearing infected cells and malignant cells in the liver, which could greatly reduce the chance of HCC [33-35]. Recent evidence also claims that NK cells kill tumor cells in cancer model [36].

Our current study demonstrated that preoperative ALRI might be a potential predictive marker for patients with HCC. According to the ROC curve, 25.2 appeared to be the most suitable reference value for preoperative ALRI with a sensitivity of $60.3 \%$ and a specificity of $67.2 \%$ for predicting HCC. Of note, such a correlation has been validated between preoperative ALRI and age, median size, TNM stage, and PVTT; all of which are linked with the progression and prognosis of HCC. 
Elevated preoperative ALRI (>25.2) was recognized as an independent prognostic factor of both poorer DFS and OS when compared to those with low preoperative ALRI $(\leq 25.2)$.

The explanation of the association between elevated preoperative ALRI and poorer prognosis of HCC patients should be further clarified. The steep rise in the serum AST is closely connected with the progression of liver diseases, which may reflect the leakage of the membrane and the damage of hepatocytes. Once the hepatic parenchymal cells are injured, intracellular AST will be released into the blood that will lead to a high concentration of serum AST. Conversely, a dramatic decrease of serum lymphocyte may impair host's antitumor immunity. CD4 ${ }^{+}$ T lymphocyte cells play a "sensing" role in detecting premalignant tumor cells and then regulate their eradication, which can hinder the occurrence and development of hepatocellular cancer [37]. An impaired functionality of dendritic cells may notably reduce the amounts of $\mathrm{CD}^{+}$ $\mathrm{T}$ lymphocyte, which may not trigger the removal of premalignant hepatocytes, resulting in a rapid development of HCC [38]. Loss of CD4 ${ }^{+} \mathrm{T}$ lymphocyte was linked with high mortality rate and reduction of survival time in HCC patients [39]. The decrease in $\mathrm{CD}^{+} \mathrm{T}$ lymphocyte correlates with the impaired activation of CTLs that may not successfully secret a series of cytotoxin performing anti-carcinogenic function within neoplastic microenvironments [40]. Apart from that, the activation of tumor-infiltrating lymphocytes significantly improves therapeutic effect in many cancers [41, 42].

Based on the results of univariate analysis, we concluded that tumor size $>5 \mathrm{~cm}$, multiple tumor number, III-IV of TNM stage, PVTT, and distant metastasis were responsible for low DFS and OS rate. This result is consistent with a previous report that multiple tumor ( $>$ three) is an unfavorable prognostic factor in HCC recurrence [43], the calculated survival rate is significantly better in HCC patients with a single tumor compared to those with multiple tumors [44], tumor number was independently associated with the survival of HCC patients [45]. PVTT is the major reason of intrahepatic metastasis, which occurs in approximately $40 \%$ of HCC patients, and is one of the most negative independent prognostic factors of OS in HCC patients. The appearance of PVTT affects liver's vascular supply, which may have considerable impact on wide dissemination of malignant tumor cells, thus severely crippling normal liver function, and resulting in poor prognosis in HCC [46-48]. There are growing evidences that the distinctive feature of an aggressive solitary HCC is its capability of metastasis, which leads to a higher possibility of HCC recurrence and is related to as much as $90 \%$ of cancer related mortality $[49,50]$.

The findings by the multivariate analysis showed that preoperative ALRI $>25.2$, III-IV of TNM stage, PVTT, and tumor size $>5 \mathrm{~cm}$ were independent prognostic markers for DFS and OS in HCC patients. This is in agreement with previous reports that tumor size may act as an independent prognostic factor for resected small HCC [51] andthe prognosis in HCC patients with tumor size $<$ $5 \mathrm{~cm}$ was better than those with tumor size $>5 \mathrm{~cm}$ [52] significantly.

Generally speaking, the TNM system has graded patients on their prognosis by using the clinical pathologic characteristics. However, it may only provide limited information for predicting patients' relapse, especially in advanced patients. In this study, we found that preoperative ALRI had favorable prognostic value for both DFS and OS in patients with TNM stage of III-IV in our cohort, which strongly substantiated preoperative ALRI could be used predicting recurrence in advanced HCC. Notably, in subgroup with tumor size smaller than $5 \mathrm{~cm}$ in diameter, preoperative ALRI > 25.2 also show its apparent prognostic value in predicting poorer DFS and OS, further supporting that preoperative ALRI may act as a potential prognostic maker for predicting survival in different HCC subgroups.

It is well known some tumor biomarkers like AFP, CEA, and CA19-9 may reflect cancer cells' growth, differentiation, invasion and metastasis to some degree $[53,54]$. However, the diagnostic sensitivity of these markers has been challenged, for example, a noticeable rise of serum AFP was not observed in $30 \%$ of $\mathrm{HCC}$ patients [55], multiple factors affected diagnostic accuracy and reliability of CEA [53], and a high level of serum CA19-9 is frequently seen in normal bile secreted by healthy biliary tract [54]. Compared to these tumor biomarkers, the ALRI test meets the requirements of highly precise diagnosis and prognosis, and reduces patients' economic cost.

We conclude that the optimal cut-off value of preoperative ALRI for predicting the prognosis of $\mathrm{HCC}$ was 25.2. Calculating preoperative ALRI is a simple method for the judgment of prognosis in HCC patients. However, our survey also has some limitations; a prospective study with a larger population should be conducted to justify our researches.

\section{MATERIALS AND METHODS}

\section{Study population}

HCC specimens, along with complete clinical and pathological data, were collected from $371 \mathrm{HCC}$ patients treated with surgical tumor resection at the Affiliated Hospital of Guilin Medical University, Guangxi, China, between March 1997 and January 2008. All the patients performed routine assessments before surgery, which included complete hematologic and biochemistry profiles, physical examination, ultrasonography (US), computed 
tomography $(\mathrm{CT})$ scans, and magnetic resonance imaging (MRI). All of the participants had no other lymphatic system disorders, such as lymphocytic leukemia and lymphoma, thus ensuring that the lymphocyte count was representative of normal baseline values. Patient baseline and clinical data, including age, gender, family history, HBsAg, alpha-fetoprotein (AFP), median size, cirrhosis, tumor number, clinical tumor-node-metastasis (TNM) stage, portal vein tumor thrombus (PVTT), distant metastasis, and recurrence are listed in table 1.

\section{Follow-up}

All 371 patients were observed regularly from the date of operation to that of death or the last follow up. Tumor recurrence was monitored by serum AFP, ultrasonography, and chest radiography every 6 months for the first two years after operation and every 3-6 months thereafter. Recurrence was diagnosed by contrast ultrasonography, MRI, and CT. The mean for the postoperative follow-up period was 36.0 months (median, 20.0 months; range, 2.0 to 84.0 months). Disease-free survival (DFS) is defined as the interval between the date of surgery and recurrence, metastasis, death or last follow-up, whereas overall survival (OS) was defined as the interval between the date of surgery and death or last follow-up.

\section{Ethics statement}

The survey protocol was in accordance with the ethical guidelines of the Declaration of Helsinki. Ethical approval was granted by the Ethical Committee of the Affiliated Hospital of Guilin Medical University, and the written consent was obtained from all examined patients or their guardians prior to surgery.

\section{Selection of cutoff score}

Receiver operating characteristic (ROC) curve analysis was utilized to determine the cutoff score of preoperative ALRI in patients with HCC. The optimal cutoff value was closest to the point with maximum sensitivity and specificity. To perform ROC curve analysis, we dichotomized the rest of clinicopathological features, and further investigated the clinicopathologic and prognostic significance of preoperative ALRI in patients with HCC.

\section{Preoperative ALRI calculation}

The standard protocols for Vitros system enzymatic rate reaction and complete fluorescent flow cytometry were used to measure the AST and lymphocyte count, respectively. The reference value of AST provided by the laboratory was $<40 \mathrm{U} / \mathrm{L}$ (male and female) [6]. Preoperative ALRI was calculated with the following formula: (AST value/ lymphocyte count) $\times 10^{9} / \mathrm{U}$.

\section{Statistical analysis}

The statistical analyses were performed using SPSS13.0 (SPSS Inc, Chicago, IL). ROC curve analysis was applied to determine the cutoff value of preoperative ALRI by 0,1 -criterion. The Pearson $\chi^{2}$ test was executed to analyze the correlation between preoperative ALRI and clinicopathological parameters. Survival curves for the HCC patients were calculated by using the Kaplan-Meier method. After the univariate analysis by the log-rank test, only variables with $p$-value $<0.05$ were utilized in the multivariate analysis, which used the Cox proportional hazards model to identify the independent prognostic factors for DFS and OS. Hazard ratios (HR) and 95\% confidence intervals (CI) were calculated. Differences were considered statically significant at $p<0.05$.

\section{ACKNOWLEDGMENTS}

The present study was supported in part by the National Natural Science Foundation of China (grant no. 81360309), the Natural Science Foundation of Guangxi (No. 2014GXNSFAA118194), the Medical Health Appropriate Technology Research and Development Project from the Health and Family Planning Commission in Guangxi (S201407-06), the Scientific Research and Technology Development Project of Guilin (201403102-1), 'Sphingolipids and Related Diseases' Program for Innovative Research Team of Guilin Medical University, and Hundred Talents Program "the Introduction of Overseas High-Level Talents in Colleges and Universities in Guangxi". This research was also supported by Guangxi Distinguished Experts Special Fund, Guangxi Health and Family Planning Commission "139" Leading Talents Training Plan, the Guangxi Culture of New Century Academic and Technical Leader of Special Funds, Scientific Research Innovation Team in Colleges and Universities of Guangxi. We thank Qining Jin (Academic Magnet High School, North Charleston, South Carolina, United States) for carefully reading the manuscript.

\section{CONFLICTS OF INTEREST}

There is no conflict of interest.

\section{REFERENCES}

1. El-Serag HB. Epidemiology of viral hepatitis and hepatocellular carcinoma. Gastroenterology. 2012; 142:1264-1273. 
2. Parkin DM, Bray F, Ferlay J, Pisani P. Global cancer statistics, 2002. CA Cancer J Clin. 2005; 55:74-108.

3. Jemal A, Bray F, Center MM, Ferlay J, Ward E, Forman D. Global cancer statistics. CA Cancer J Clin. 2011; 61:69-90.

4. Danese E, Montagnana M, Minicozzi AM, Bonafini $\mathrm{S}$, Ruzzenente $\mathrm{O}$, Gelati $\mathrm{M}$, et al. The role of resistin in colorectal cancer. Clin Chim Acta. 2012; 413:760-764.

5. Zhang GM, Xia YJ, Guo XX, Zhu BL, Zhang GM, Ma XB, et al. Reference intervals for total bilirubin, ALT, AST and creatinine in healthy Chinese elderly. Med Sci Monit. 2014; 20:1778-1782.

6. van Beek JH, de Moor MH, de Geus EJ, Lubke GH, Vink JM, Willemsen G, et al. The genetic architecture of liver enzyme levels: GGT, ALT and AST. Behav Genet. 2013; 43:329-339.

7. Lee TH, Kim WR, Benson JT, Therneau TM, Melton LJ 3rd. Serum aminotransferase activity and mortality risk in a United States community. Hepatology. 2008; 47:880-887.

8. Witjes CD, IJzermans JN, van der Eijk AA, Hansen BE, Verhoef C, de Man RA. Quantitative HBV DNA and AST are strong predictors for survival after $\mathrm{HCC}$ detection in chronic HBV patients. Neth J Med. 2011; 69:508-513.

9. Grivennikov SI, Greten FR, Karin M. Immunity, inflammation, and cancer. Cell. 2010; 140:883-899.

10. Coussens LM, Werb Z. Inflammation and cancer. Nature. 2002; 420:860-867.

11. Tarao K, Miyakawa K, Miyagi Y, Ohkawa S, Morinaga $\mathrm{S}$, Oshige K, et al. Severe inflammation in the background liver cirrhosis correlates with the development of poorly differentiated $\mathrm{HCC}$ in $\mathrm{HCV}$-associated liver cirrhosis. Intern Med. 2012; 51:2495-2501.

12. Chen L, Zhang Q, Chang W, Du Y, Zhang H, Cao G. Viral and host inflammation-related factors that can predict the prognosis of hepatocellular carcinoma. Eur J Cancer. 2012; 48:1977-1987.

13. Mantovani A, Allavena P, Sica A, Balkwill F. Cancerrelated inflammation. Nature. 2008; 454:436-444.

14. Ko E, Jung G. Positive association of long telomeres with the invasive capacity of hepatocellular carcinoma cells. Biochem Biophys Res Commun. 2014; 447:358-363.

15. Mossanen JC, Tacke F. Role of lymphocytes in liver cancer. Oncoimmunology. 2013; 2:e26468. [PubMed: 24498546]

16. Shibolet O, Alper R, Zlotogarov L, Thalenfeld B, Engelhardt D, Rabbani E, et al. NKT and CD8 lymphocytes mediate suppression of hepatocellular carcinoma growth via tumor antigen-pulsed dendritic cells. Int J Cancer. 2003; 106:236-243.

17. Guo CL, Yang HC, Yang XH, Cheng W, Dong TX, Zhu WJ, et al. Associations between infiltrating lymphocyte subsets and hepatocellular carcinoma. Asian Pac J Cancer Prev. 2012; 13:5909-5913.

18. Okuda M, Li K, Beard MR, Showalter LA, Scholle F, Lemon SM, et al. Mitochondrial injury, oxidative stress, and antioxidant gene expression are induced by hepatitis $\mathrm{C}$ virus core protein. Gastroenterology. 2002; 122:366-375.

19. Yamashita Y, Shirabe K, Toshima T, Tsuijita E, Takeishi K, Harimoto $\mathrm{N}$, et al. Risk factors for recurrence after curative resection of hepatitis C-related hepatocellular carcinoma in patients without postoperative interferon therapy. Hepatol Res. 2013; 43:1313-1320.

20. Lin YJ, Lee MH, Yang HI, Jen CL, You SL, Wang LY, et al. Predictability of liver-related seromarkers for the risk of hepatocellular carcinoma in chronic hepatitis B patients. PLoS One. 2013; 8:e61448.

21. Mera JR, Dickson B, Feldman M. Influence of gender on the ratio of serum aspartate aminotransferase (AST) to alanine aminotransferase (ALT) in patients with and without hyperbilirubinemia. Dig Dis Sci. 2008; 53:799-802.

22. Hann HW, Wan S, Lai Y, Hann RS, Myers RE, Patel F, et al. AST to platelet ratio index as a prospective predictor of hepatocellular carcinoma risk in patients with chronic HBV infection. J Gastroenterol Hepatol. 2015; 30:131-138.

23. Shen SL, Fu SJ, Chen B, Kuang M, Li SQ, Hua YP, et al. Preoperative Aspartate Aminotransferase to Platelet Ratio is an Independent Prognostic Factor for Hepatitis B-Induced Hepatocellular Carcinoma After Hepatic Resection. Ann Surg Oncol. 2014; 21:3802-3809.

24. Lin ZH, Xin YN, Dong QJ, Wang Q, Jiang XJ, Zhan SH, et al. Performance of the aspartate aminotransferase-to-platelet ratio index for the staging of hepatitis C-related fibrosis: an updated meta-analysis. Hepatology. 2011; 53:726-736.

25. Borsoi Viana MS, Takei K, Collarile Yamaguti DC, Guz B, Strauss E. Use of AST platelet ratio index (APRI Score) as an alternative to liver biopsy for treatment indication in chronic hepatitis C. Ann Hepatol. 2009; 8:26-31.

26. El-Serag HB, Rudolph KL. Hepatocellular carcinoma: epidemiology and molecular carcinogenesis. Gastroenterology. 2007; 132:2557-1576. ]

27. Pagès F, Galon J, Dieu-Nosjean MC, Tartour E, SautèsFridman C, Fridman WH. Immune infiltration in human tumors: a prognostic factor that should not be ignored. Oncogene. 2010; 29:1093-1102.

28. Chew V, Chen J, Lee D, Loh E, Lee J, Lim KH, et al. Chemokine-driven lymphocyte infiltration: an early intratumoural event determining long-term survival in resectable hepatocellular carcinoma. Gut. 2012; 61:427438.

29. Schneider C, Teufel A, Yevsa T, Staib F, Hohmeyer A, Walenda $\mathrm{G}$, et al. Adaptive immunity suppresses formation and progression of diethylnitrosamine-induced liver cancer. Gut. 2012; 61:1733-1743.

30. Qin Z, Schwartzkopff J, Pradera F, Kammertoens T, Seliger $\mathrm{B}$, Pircher H, et al. A critical requirement of interferon gamma-mediated angiostasis for tumor rejection by CD8+ T cells. Cancer Res. 2003; 63:4095-4100.

31. Tosolini M, Kirilovsky A, Mlecnik B, Fredriksen T, Mauger $\mathrm{S}$, Bindea $\mathrm{G}$, et al. Clinical impact of different classes of infiltrating $\mathrm{T}$ cytotoxic and helper cells (Th1, th2, treg, 
th17) in patients with colorectal cancer. Cancer Res. 2011; 71:1263-1271.

32. Zimmermann HW, Tacke F. Modification of chemokine pathways and immune cell infiltration as a novel therapeutic approach in liver inflammation and fibrosis. Inflamm Allergy Drug Targets. 2011; 10:509-536.

33. Tian Z, Chen Y, Gao B. Natural killer cells in liver disease. Hepatology. 2013; 57(4):1654-1662. [PubMed: 23111952]

34. Subleski JJ, Wiltrout RH, Weiss JM. Application of tissue-specific NK and NKT cell activity for tumor immunotherapy. J Autoimmun. 2009; 33:275-281.

35. Cai L, Zhang Z, Zhou L, Wang H, Fu J, Zhang S, et al. Functional impairment in circulating and intrahepatic NK cells and relative mechanism in hepatocellular carcinoma patients. Clin Immunol. 2008; 129:428-437.

36. Stojanovic A, Cerwenka A. Natural killer cells and solid tumors. J Innate Immun. 2011; 3:355-364.

37. Kang TW, Yevsa T, Woller N, Hoenicke L, Wuestefeld T, Dauch D, et al. Senescence surveillance of pre-malignant hepatocytes limits liver cancer development. Nature. 2011; 479:547-551.

38. Ormandy LA, Farber A, Cantz T, Petrykowska S, Wedemeyer H, Horning M, et al. Direct ex vivo analysis of dendritic cells in patients with hepatocellular carcinoma. World J Gastroenterol. 2006; 12:3275-3282.

39. Fu J, Zhang Z, Zhou L, Qi Z, Xing S, Lv J, et al. Impairment of CD4+ cytotoxic $T$ cells predicts poor survival and high recurrence rates in patients with hepatocellular carcinoma. Hepatology. 2013; 58:139-149.

40. Witkowski M, Spangenberg HC, Neumann-Haefelin C, Büttner N, Breous E, Kersting N, et al. Lack of ex vivo peripheral and intrahepatic alpha-fetoprotein-specific CD4+ responses in hepatocellular carcinoma. Int J Cancer. 2011; 129:2171-2182.

41. Baitsch L, Baumgaertner P, Devêvre E, Raghav SK, Legat A, Barba L, et al. Exhaustion of tumor-specific CD8(+) T cells in metastases from melanoma patients. J Clin Invest. 2011; 121:2350-2360.

42. Klebanoff CA, Acquavella N, Yu Z, Restifo NP. Therapeutic cancer vaccines: are we there yet? Immunol Rev. 2011; 239:27-44.

43. Chan KM, Chou HS, Wu TJ, Lee CF, Yu MC, Lee WC. Characterization of hepatocellular carcinoma recurrence after liver transplantation: perioperative prognostic factors, patterns, and outcome. Asian J Surg. 2011; 34:128-134.

44. Tandon P, Garcia-Tsao G. Prognostic indicators in hepatocellular carcinoma: a systematic review of 72 studies. Liver Int. 2009; 29:502-510.

45. Yeh CT, Huang YH, Liang KH, Chang ML, Hsu CW, Chen $\mathrm{YC}$, et al. Segregation of signaling proteins as prognostic predictors for local recurrence and distant metastasis in hepatocellular carcinoma. Int J Oncol. 2014; 44:491-504.

46. Minagawa M, Makuuchi M. Treatment of hepatocellular carcinoma accompanied by portal vein tumor thrombus.
World J Gastroenterol. 2006; 12:7561-7567.

47. Cabibbo G, Enea M, Attanasio M, Bruix J, Craxì A, Cammà C. A meta-analysis of survival rates of untreated patients in randomized clinical trials of hepatocellular carcinoma. Hepatology. 2010; 51:1274-1283.

48. Lau WY, Sangro B, Chen PJ, Cheng SQ, Chow P, Lee $\mathrm{RC}$, et al. Treatment for hepatocellular carcinoma with portal vein tumor thrombosis: the emerging role for radioembolization using yttrium-90. Oncology. 2013; 84:311-318.

49. Portolani N, Coniglio A, Ghidoni S, Giovanelli M, Benetti A, Tiberio GA, et al. Early and late recurrence after liver resection for hepatocellular carcinoma: prognostic and therapeutic implications. Ann Surg. 2006; 243:229-235.

50. Chaffer CL, Weinberg RA. A perspective on cancer cell metastasis. Science. 2011; 331:1559-1564.

51. Chen YL, Ko CJ, Chien SY, Chen LS, Chen ML, Chi $\mathrm{CW}$, et al. Tumor size as a prognostic factor in resected small hepatocellular carcinoma: a controversy revisited. J Gastroenterol Hepatol. 2011; 26:851-857.

52. Hoshida Y, Villanueva A, Kobayashi M, Peix J, Chiang DY, Camargo A, et al. Gene expression in fixed tissues and outcome in hepatocellular carcinoma. N Engl J Med. 2008; 359:1995-2004.

53. Li Y, Li DJ, Chen J, Liu W, Li JW, Jiang P, Zhao X, Guo F, Li XW, Wang SG, et al. Application of Joint Detection of AFP, CA19-9, CA125 and CEA in Identification and Diagnosis of Cholangiocarcinoma. Asian Pac J Cancer Prev. 2015;16:3451-3455.

54. Chen YL, Chen $\mathrm{CH}, \mathrm{Hu} \mathrm{RH}, \mathrm{Ho} \mathrm{MC}$, Jeng YM, et al. Elevated Preoperative Serum CA19-9 Levels in Patients with Hepatocellular Carcinoma Is Associated with Poor Prognosis after Resection. Scientific World Journal. 2013; 2013:380797.

55. Bialecki ES, Di Bisceglie AM. Diagnosis of hepatocellular carcinoma. HPB: the official journal of the International Hepato Pancreato Biliary Association. 2005; 7:26-34. 